3-31-2018

\title{
Testing Memory Forensics Tools for the Macintosh OS X Operating System
}

\author{
Charles B. Leopard \\ U.S. Naval Postgraduate School, cbleopard@gmail.com \\ Neil C. Rowe \\ U.S. Naval Postgraduate School, ncrowe@nps.edu \\ Michael R. McCarrin \\ mrmccarr@nps.edu
}

Follow this and additional works at: https://commons.erau.edu/jdfsl

Part of the Computer Law Commons, and the Information Security Commons

\section{Recommended Citation}

Leopard, Charles B.; Rowe, Neil C.; and McCarrin, Michael R. (2018) "Testing Memory Forensics Tools for the Macintosh OS X Operating System," Journal of Digital Forensics, Security and Law. Vol. 13 : No. 1 , Article 7.

DOI: https://doi.org/10.15394/jdfsl.2018.1491

Available at: https://commons.erau.edu/jdfsl/vol13/iss1/7

This Article is brought to you for free and open access by the Journals at Scholarly Commons. It has been accepted for inclusion in Journal of Digital Forensics, Security and Law by an authorized administrator of Scholarly Commons. For more information, please contact commons@erau.edu.

(c)ADFSL 


\section{Testing Memory Forensics Tools for the Macintosh OS X Operating System}

\section{Cover Page Footnote}

The views expressed are those of the authors and do not represent the U.S. Government. A shortened version of this paper appeared in the Proceedings of the Ninth EAI International Conference on Digital Forensics and Computer Crime, Prague, Czech Republic, October 2017. 


\title{
TESTING MEMORY FORENSICS TOOLS FOR THE MACINTOSH OS X OPERATING SYSTEM
}

\author{
Charles B. Leopard, Neil C. Rowe, and Michael R. McCarrin \\ U.S. Naval Postgraduate School, Computer Science Department \\ Monterey, California, 93940 US \\ (831) 656-2462, fax (831) 656-2814 \\ cbleopard@gmail.com, ncrowe@nps.edu, mrmccarr@nps.edu
}

\begin{abstract}
Memory acquisition is essential to defeat anti-forensic operating-system features and investigate cyberattacks that leave little or no evidence in secondary storage. The forensic community has developed tools to acquire physical memory from Apple's Macintosh computers, but they have not much been tested. This work tested three major OS X memory-acquisition tools. Although the tools could capture system memory accurately, the open-source tool OSXPmem appeared advantageous in size, reliability, and support for memory configurations and versions of the OS X operating system.
\end{abstract}

Keywords: digital forensics; acquisition; main memory; Macintosh; OSX; testing

\section{INTRODUCTION}

As Recent Macintosh OS X operating systems incorporate many anti-forensic features, most notably in cloud storage and encryption. Users can fully encrypt many things, including entire volumes of the secondary storage, making it impossible to recover forensic evidence from storage in a reasonable period of time without passwords. Because of this, forensics on the main memory of such systems is increasingly valuable. Memory forensics can recover running processes, network packets, communications artifacts, encryption keys, and injected code from volatile memory. It is helpful to compare available tools for memory forensics.

\section{PREVIOUS WORK}

Memory acquisition is a challenge for digital forensics because memory is volatile, and a tool must interact gracefully with an operating system, as noted in Sutherland et al. (2008). Errors can occur with any memory acquisition because memory changes unpredictably over time, as discussed in Pan et al. (2008), but tools can do several things to minimize errors. While there are a number of memoryacquisition tools and analysis programs for Windows operating systems (see Neethu et al., 2014) and Android devices (see Li et al., 2016), there are only a few for Macintosh systems (see Ligh et al., 2014). Macintosh machines generally impose stiffer access controls than Windows machines, but they are more aggressive in caching, which permits recovering some main memory even when the machine has been turned off, as noted by BlackBag Technologies (2017). 
Most research on memory acquisition has focused on developing new techniques, with only a few projects testing tools. Carvajal et al. (2013) compared six Windows tools in their ability to extract memory quickly without leaving artifacts. Kamal et al. (2016) also tested six Windows tools (two different from the previous work) and focused on the artifacts left by the tools. Ahmed and Aslam (2015) compared six Windows tools (four different from Carvajal et al) and found a few differences in performance between them, especially when anti-forensics measures were taken on the target machines.

The abovementioned tools provide software-based acquisition, in contrast to tools such as Inception and Passware's Firewire Memory Imager that are hardware-supported. Hardware support can circumvent password protection, but there must be different tool versions for different hardware and they can be limited in how much memory they can acquire. Modern software-based tools generally require a kernel to be installed on the target system, must deal with memory compression, and must be careful to avoid interfering with reserved areas of memory as discussed in Libser and Kornblum (2008). Memory-acquisition tools can be distinguished from memory-analysis tools such as the open-source Volatility (2015) and Rekall (2015) that allow inspection of specific artifacts, and analysis tools require detailed knowledge of where an operating system keeps things, so they require frequent updates.

For Macintosh machines, software-based acquisition tools available are MacQuisition (www.blackbagtech.com/software-

products/macquisition.html), Google/Rekall OSXPMem (releases.rekall-forensic.com), Sumuri Forensics RECON (sumueri.com/software/recon), Mandiant Mac Memoryze (www.fireeye.com/ services/freeware/memoryze.html), and
Volafox Volatility (code.google.com/p/volafox). (www.vmware.com/products/fusion.html) after version 10.7 also provide some Macintosh memory-forensic capabilities.

\section{METHODOLOGY}

This work tested the three Macintosh memoryacquisition tools: MacQuisition version 2014R1, OSXPMem version RC3, and Sumuri Forensics RECON version 1.0.11. We evaluated the tools on (1) ability to write a physical-memory capture without crashing the target computer system, (2) obtrusiveness (what its memory footprint was and how long it took to run), and (3) ability to produce a capture from which standard forensic artifacts could be recovered using the Volatility Framework and the Rekall Memory Forensic Framework. The Rekall plugins used were arp (ARP tables), ifconfig (network interface), lsof (open files), mount (mount points), netstat (network connections), psaux (processes), and route (routing tables). The Volatility plugins used were mac_arp, mac_bash (Bash shell history), mac-ifconfig, mac_lsof, mac-mount, mac_netstat, mac_psaux, and mac_route. We were particularly interested in comparing memory snapshots of tools in similar states since they could indicate functional differences or coverage gaps.

This work first tested the OSX Mavericks (10.9.5) operating system, and then retested after upgrading to Yosemite 10.10.1 and 10.10.2 (more details are given in Leopard, 2015). Each tool was directed to write a memory capture to an external USB 3 hard drive of 7200RPM. In total, 450 captures were performed: 50 machines, 3 operating systems, and 3 forensic tools.

We did five kinds of experiments: (1) testing the tools' ability to recover memory, (2) testing the localizing of encryption keys, (3) observing memory changes over time, (4) 
comparing outputs between tools, and (5) comparing with output for a non-virtual environment. In all these experiments, we ran the operating systems with no additional modifications, software, or tasks.

The rate at which memory changes affects the memory dumps acquired by tools. To analyze the systems more accurately, as well obtain flexibility in the setup, we created a virtual machine as in Zhang et al. (2010). We used VMware Fusion Professional version 7.1.1 and took a series of snapshots. The virtual machine ran the Mavericks operating system and was configured to use two processor cores and $8 \mathrm{GiB}$ of memory. The host machine was a MacBook Pro (Retina, 15-inch, 2014) with a $2.8 \mathrm{GHz}$ Intel i7 processor and $16 \mathrm{GiB}$ of 1600 $\mathrm{MHz}$ DDR3 memory. Our specific procedure was: (1) $\log$ into the virtual machine and take a snapshot every minute for the first fifteen minutes, and then at 20, 25, and 30 minutes; (2) use the Volatility plugin to decompress the snapshots; (3) create MD5 hashes for every 4 $\mathrm{KiB}$ block; and (4) compare the MD5 hashes with the original hashes and note differences. We compared memory captured by each of our tools against the virtual machine snapshot that completed at a time closest to when each capture completed. We then compared the memory captures taken with the tools.

Memory acquisition can be assessed in terms in its correctness (whether it captures what is stored in memory at some time), its atomicity (whether it shows concurrent activity), and its integrity (its persistence in correctness for some guaranteed time period), as suggested in Vömel and Freiling (2012). The current work addressed correctness and (in a simple way) integrity, but not atomicity since analyzing that requires more precise tools.

\section{RESULTS}

\subsection{Ability to Recover Memory}

All three tools successfully acquired memory. Acquisition speeds were within $7 \%$ for the three. Physical memory sizes were $67.45 \mathrm{MiB}$ for MacQuisition, $0.944 \mathrm{MiB}$ for OSXPmem, and 206.7 MiB for RECON. Shared memory and private memory sizes were proportional. OSXPmem had a size advantage as a command-line tool without a graphical user interface.

We observed several crashes caused by tools. Valuable forensic data can be permanently lost in a crash, so crash danger is important. No machine crashed more than once, and after a crash, a second acquisition attempt was often successful after the machines restarted, with one exception. The exception was when RECON was used to acquire memory from the Mac Pros with 64 GiB of RAM; all the machines crashed, and additional attempts also failed. Nonetheless, examination of the results confirmed that if a memory capture was completed without a crash, the capture contained all forensic artifacts found by the other tools.

\subsection{Localizing Encryption Keys}

The Passware Password Recovery Kit Forensic Version 13.1 was used to confirm that encryption keys for FileVault2 were located within the OS X memory captures and could be used to decrypt the volume. To do this, FileVault2 was enabled on a MacBook Pro and a Mac Pro computer running Mavericks as well as on a MacBook Pro and a Mac Pro computer running Yosemite. Memory captures were done with MacQuisition, OSXPMem, and RECON on both the MacBook Pro and Mac Pro computers running Mavericks. RECON failed to capture physical memory from the Mac Pro computers. OSXPMem was used to capture memory from the Mac Pro and MacBook Pro 
running Yosemite. MacQuisition did not support Yosemite at the time.

Passware located the encryption keys in all of the memory captures and successfully decrypted the FileVault2 volumes. The OS X user's login password was located within all the memory captures using the hex editor iBored by searching for the term "longname" which we found frequently near a user's password. The password remained in the same block of memory during the thirty-minute period on all captures.

\subsection{Observing Memory Changes over Time}

Memory captures (snapshots) were taken over a period on a virtual machine with Mac OS version Mavericks installed. A Python script counted the $4 \mathrm{KiB}$ blocks whose hash values changed from the original snapshot. Typically, the virtual machine used $1.35 \mathrm{GiB}$ of memory ran 110-120 processes during tests. Results showed that on average only $5.33 \%$ of the blocks had changed after 30 minutes when running only default processes, and not smoothly (Figure 1).

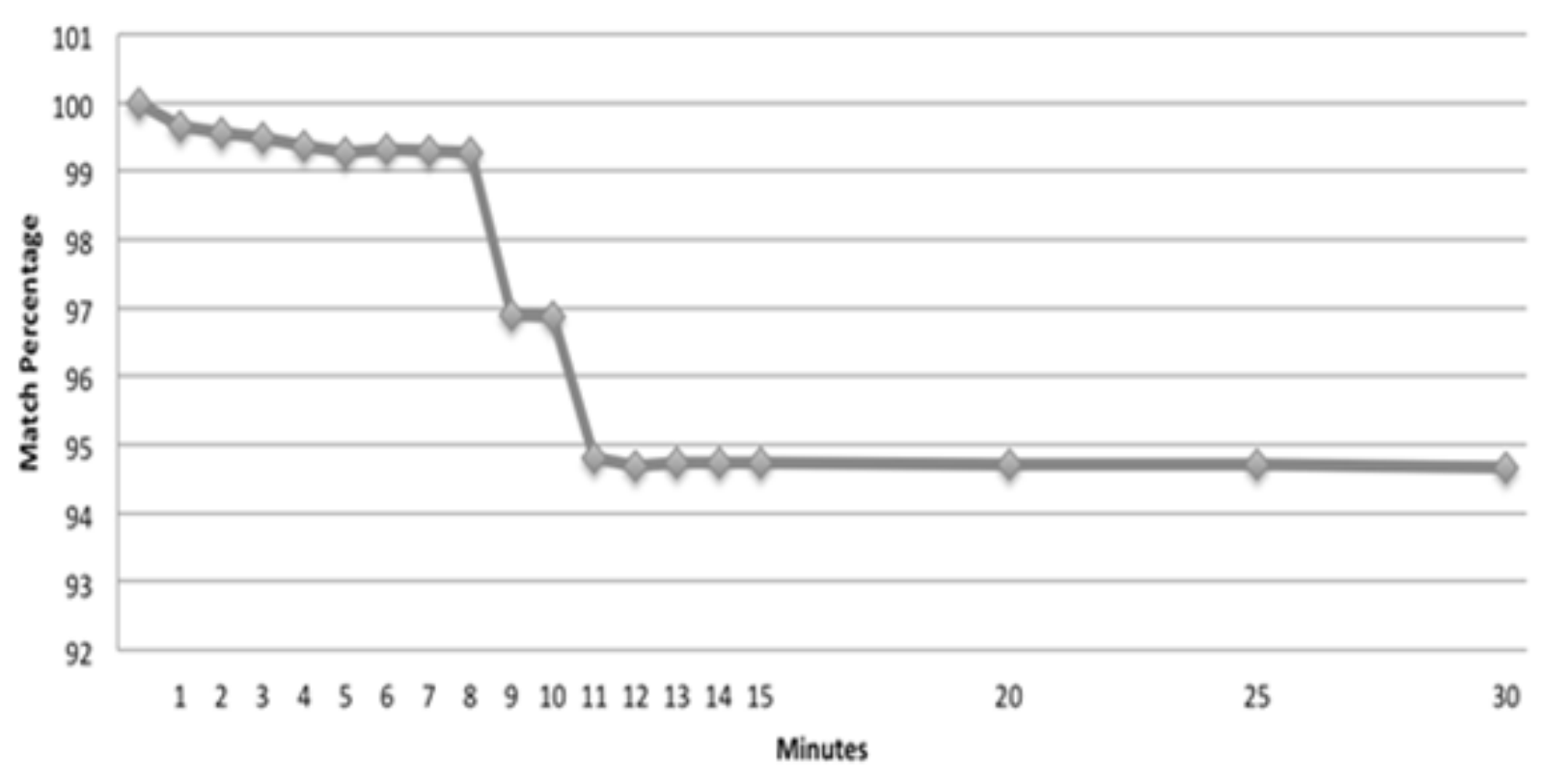

Figure 1. Average block hash match percentage over time on OSX Mavericks.

Memory captures were larger than the allocated physical memory due to the presence of reserved areas. The datasheet for Fourth Generation Intel Core Processor Address Map describes reserved areas below $4 \mathrm{GiB}$ that do not belong to the dynamic random-access memory (see Intel, 2012). Because of this, when the vmem files containing the memory in the virtual-machine snapshots were converted to raw images, each vmem file was $9 \mathrm{GiB}$ in size, though the virtual-machine configuration allocated $8 \mathrm{GiB}$ to physical memory. Stuttgen and Cohen (2015) discuss how physical memory addresses are used for communication with devices (video cards, PCI cards, and flash memory) on the motherboard with memorymapped I/O. The $1 \mathrm{GiB}$ block ranges observed between $3 \mathrm{GiB}$ and $4 \mathrm{GiB}$ appear to be 
reserved for this, and the chipset routes memory access around these reserved regions.

\subsection{Comparing Tools}

All three tools captured the same range of null values in the first half of the memory graphs. The MacQuisition device log did report "bad addresses" were padded with zeroes beginning at block 786432 and ending at block 1048575 . Each block contained 4096 bytes resulting in approximately $1 \mathrm{GiB}$ of null characters. We inspected the block range in all three tool memory captures with a hex editor and confirmed that all the tools padded the same block range with zeroes.

Figure 2 shows example 4-KiB block matches between the tool-acquired memory captures of the virtual machine and the virtual-machine snapshot SV1 taken 1 minute after start. Figure 3 shows matches among the tool images themselves at the same times. The vertical axis represents slices of $4 \mathrm{MiB}$, so each diagram shows around $9 \mathrm{GiB}$ of memory. The three plots in both Figures show MacQuisition, OSXPmem, and RECON in order. The darker areas represent matches to the initial memory state without null (zero) characters; grey blocks represent blocks that match but contain null characters; and the white blocks represent blocks that have changed and do not match. The top of the diagram represents the beginning of memory. The waviness of the boundaries is intended to make them easier to see and does not indicate uncertainty.

Overall, we saw similar regions of non-null matches (the white areas) in all comparisons. However, the tool memory captures showed that most of the null characters observed in the virtual-machine snapshots were overwritten with data when the acquisition tools themselves acquired the memory. This would suggest as the memory-capture tools run, blocks of memory containing null characters get changed while other blocks remain mostly unchanged. Since these regions are large and outside the memory space used by the tools, it is unlikely that the tools themselves are changing the data directly. Rather, we conclude that some other mechanism of the operating system is writing to unused space in memory during the acquisition process. 

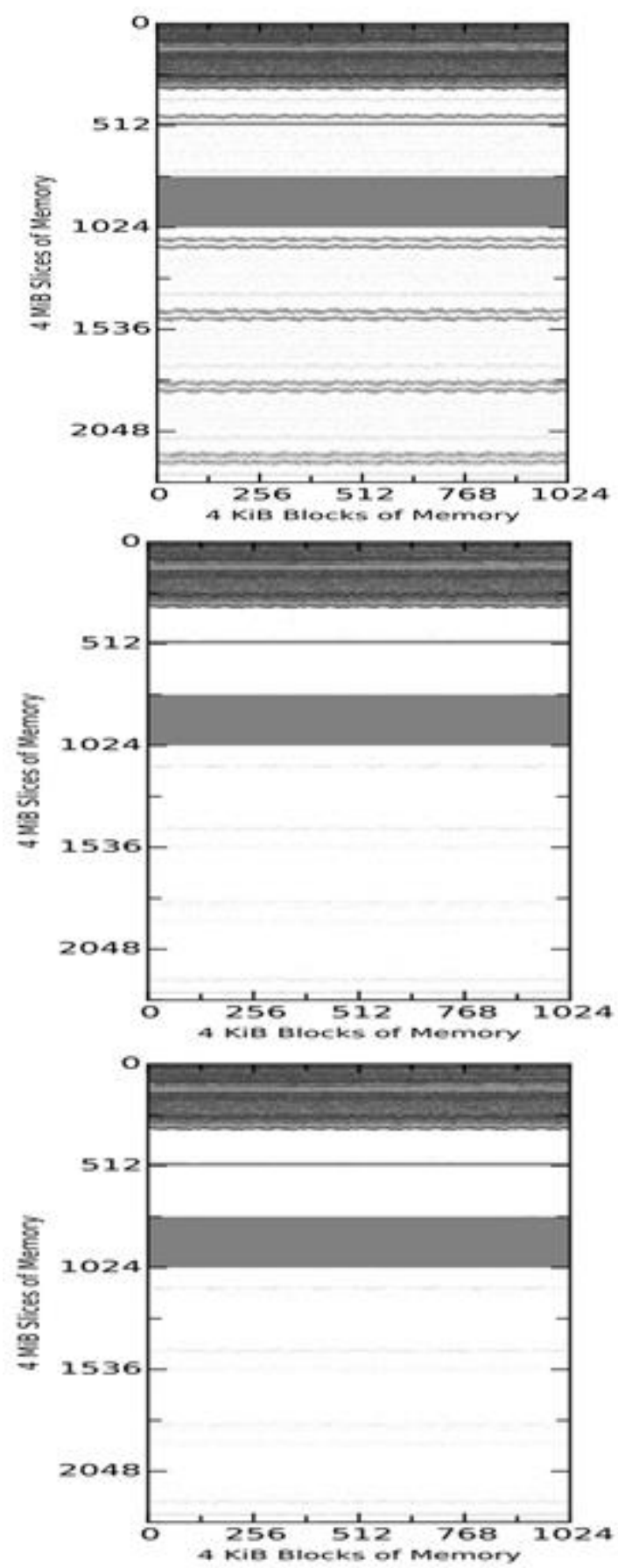

Figure 2. Virtual Machine Comparisons of MacQuisition (Upper Left), OSXPmem (Upper Right), and RECON (Lower Left) to the Snapshot after 1 Minute on Each Machine (Vertical Axis Measures 4MiB Slices) 

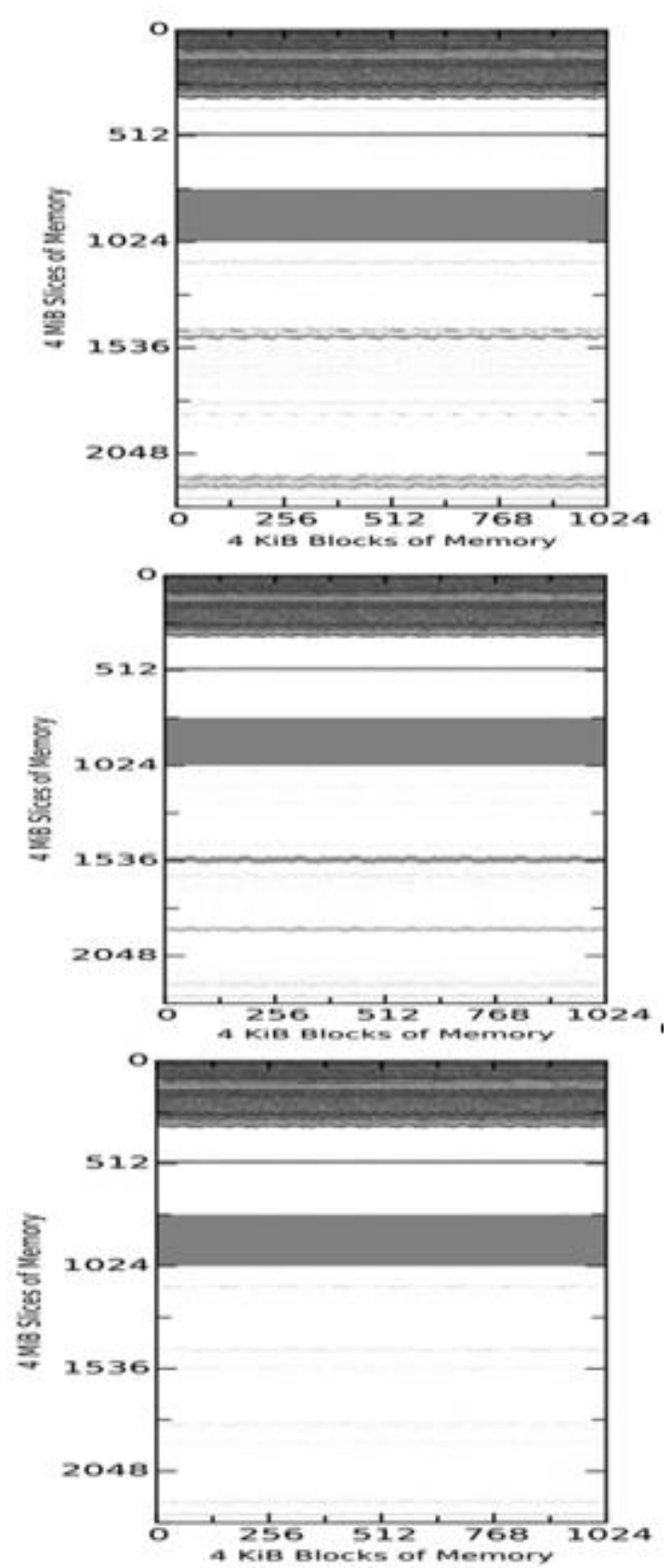

Figure 3. Comparison of the Three Tools on Analogous States of a Virtual Machine

Figure 3 also shows that each tool is capturing the same matched blocks and the same reserved region as the virtual-machine snapshot. But the tools differ in how they capture blocks of memory that do not match the blocks from snapshot. This suggests not only that the tools are introducing change to the memory space during the acquisition process, but also that each is changing the space in a unique way, so the changes from different tools do not match each other. Nonetheless, we saw no evidence of 
randomizing of memory locations on reboots, as described in $\mathrm{Gu}$ and Lin (2016), at this level of granularity.

\subsection{Comparing to a Non-Virtual Environment}

To control for possible effects due solely to VMware's environment, we tested the tools in a non-virtual environment of a physical Mac Mini (late 2014) with a $2.6 \mathrm{GHz}$ Intel i7 processor, $8 \mathrm{GiB}$ of $1600 \mathrm{MHz}$ DDR3 memory, and running Mavericks. Each tool acquired a 9.74 GiB raw file (Figure 4). There were nonmatching blocks in the first $2 \mathrm{GiB}$ of the memory that had not been observed before, but they were not clustered, causing the gray shading. All tools captured the same range of null values $2 \mathrm{GiB}$ to $4 \mathrm{GiB}$ with some small differences in the claimed boundaries, and overall this reserved area appeared to be larger than in the virtual-machine memory images. Analysis with a hex editor determined that the reserved region began at $2.17 \mathrm{GiB}$ and continued until $4 \mathrm{GiB}$. It is clear there are more changes to memory on a real machine.

Data also showed that the block matches containing non-null characters were initially around $30 \%$ of the total captured material, but remained relatively stable, declining by less than $10 \%$ over a 30 -minute period (Figure 5 ). The match percent was less than what was observed in the virtual environment as Figure 3 also suggests.

\section{CONCLUSION}

MacQuisition, RECON, and OSXPmem were all successful in capturing memory from OSX on Macintosh computers. However, OSXPmem bettered its proprietary counterparts in its size, reliability, and support for memory configurations and versions of the OSX operating system. Volatility and Rekall could use the memory captures from all three tools to obtain artifacts such as FileVault2 encryption keys. Nonetheless, we observed crashes. Ligh et al. (2015) acknowledges that all memory-acquisition tools can cause system crashes since the tool may access a reserved region at the wrong time or interfere with a system-critical function. Interference may be hard to anticipate because of variations between OSX versions and installed hardware. 

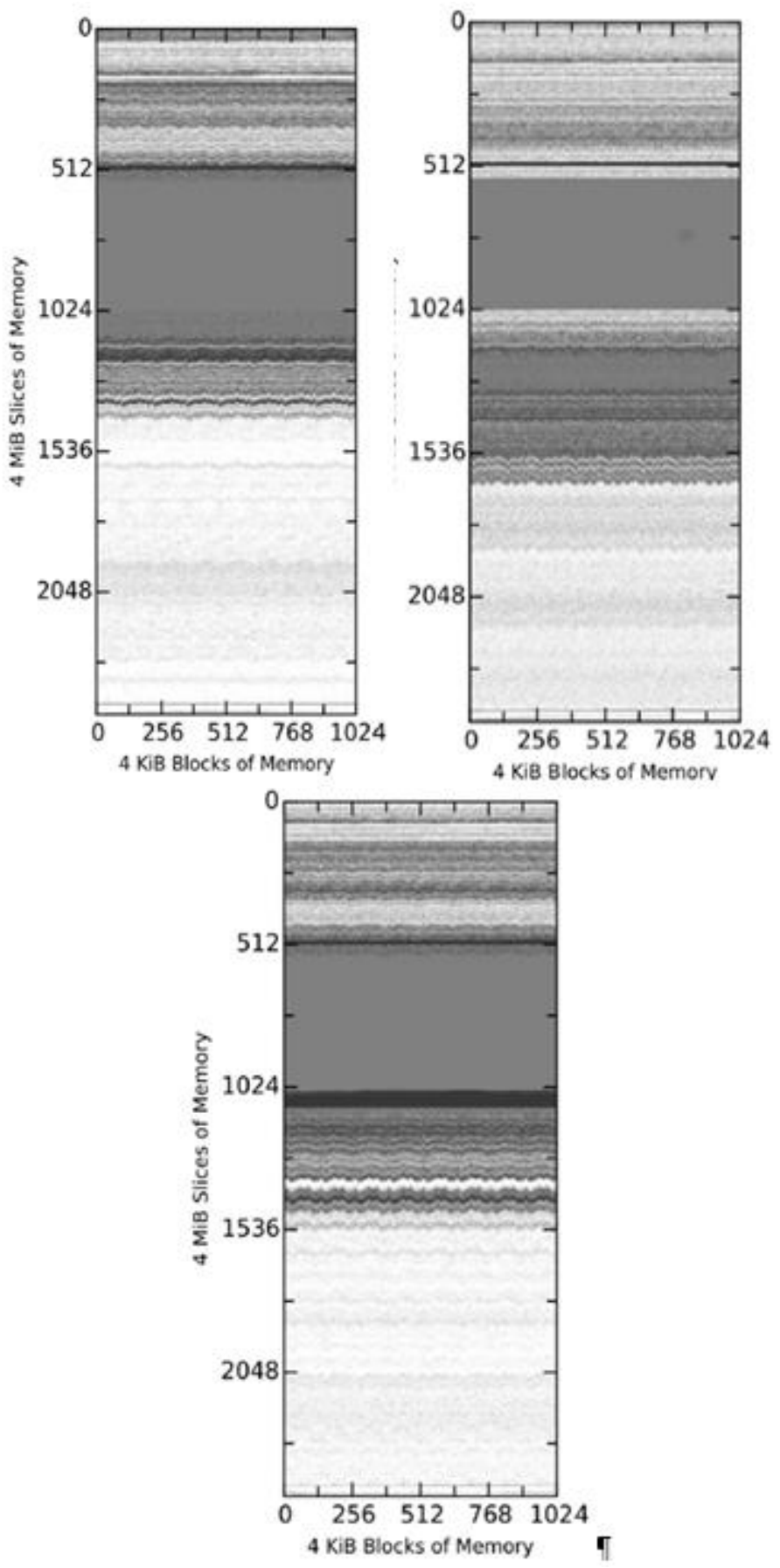

Figure 4. Comparison of Snapshots on a Real Machine over 30 Minutes 


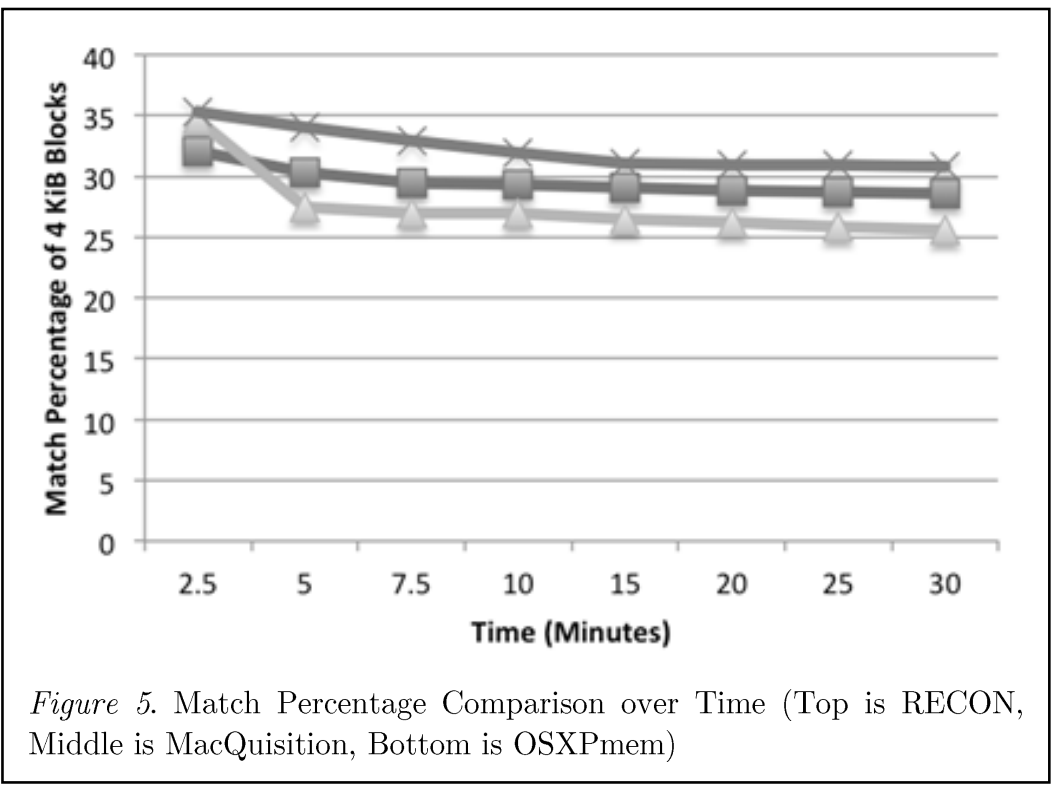

Our results showed that size of the memory capture was constant over the tools. Memory dumps were larger than the amount of physical memory (17.99 GiB versus $16 \mathrm{GiB}$ for MacBook Pro was typical) due to reserved regions.

Comparison of the virtual-machine snapshots taken over thirty minutes showed that with running only the default processes, memory did not change much. Though significant regions of memory did not match between the tool-acquired dumps and the virtual-machine snapshots, these regions contained nulls in the baseline snapshot. Because of this, our analysis of forensic artifacts using Volatility and Rekall failed to detect any situations in which the nonmatching regions corresponded to a loss of forensic evidence.

The experiments with a non-virtual environment showed the memory captures from all three tools appeared similar in the blocks that matched but did not match as well for the blocks containing null characters. The results also agree with the results of our tests in the virtual environment in that the regions of memory that match between comparisons did not change much over time.

Future work will examine in more detail the exact changes in files over time and the discrepancies between different tools. Discrepancies suggest, without having to analyze the operating system, where volatile memory stores key operating-system parameters and links. Future work will also investigate the effects of simultaneously running additional software on the operatingsystem memory images.

\section{ACKNOWLEDGEMENTS}

The views expressed are those of the authors and do not represent the U.S. Government. A shortened version of this paper appeared in the Proceedings of the Ninth EAI International Conference on Digital Forensics and Computer Crime, Prague, Czech Republic, October 2017. 


\section{REFERENCES}

Ahmed W., Aslam B. (2015). A Comparison of Windows Physical Memory Acquisition Tools. Proc. IEEE Military Communications Conference, Tampa, Florida, US, October, pp. 1292-1297.

BlackBag Technologies. (2017). Mac RAM Imaging and Analysis. Retrieved November 14, 2017 from www.blackbagtech.com/blog/ 2017/02/24/mac-ram-imaging-analysis, February 24.

Carvajal L., Varol C., Chen L. (2013). Tools for Collecting Volatile Data: A Survey Study. Proc. IEEE Conf. on Technological Advances in Electrical, Electronics, and Computer Engineering, Konya, Turkey, May.

Gu Y., Lin Z. (2016). Derandomizing Kernel Address Space Layout for Memory Introspection and Forensics. Proc. Sixth ACM Conf. on Data and Application Security and Privacy, New Orleans, Louisiana, US, March, pp. 62-72.

Intel Corporation. (2012). Desktop 4th Generation Intel Core Processor Family, Desktop Intel Pentium Processor Family, and Desktop Intel Celeron Processor Family. Retrieved from May 25, 2015 from www.intel.com/ content/dam/www/public/us/en/documen ts/datasheets/4th-gen-core-family-desktopvol-2-datasheet.pdf.

Kamal K., Alfadel M., Munia M. (2016). Memory Forensics Tools: Comparing Time and Left Artifacts on Volatile Memory. Proc. Intl. Workshop on Computational Intelligence, Dhaka, Bangladesh, December, pp. 84-90.
Leopard, C. (2015). Memory Forensics and the Macintosh OS X Operating System. M.S. thesis, U.S. Naval Postgraduate School, June. Retrieved June 15, 2015 from faculty.nps.edu/ ncrowe/oldstudents/cleopard_thesis.htm.

Li Z., Xi B., and Wu S. (2016). Digital Forensics and Analysis for Android Devices. Proc. 11th Intl. Conf. on Computer Science and Education, Nagoya, Japan, August, pp. 496-500.

Libser E., Kornblum J. (2008) A Proposal for an Integrated Memory Acquisition Mechanism. ACM SIGOPS Operating Systems Review, Vol. 42, No. 3, April, pp. 14-20.

Ligh M., Case A., Levy J., Walters A. (2014). Art of Memory Forensics. Indianapolis, IN: Wiley.

Neetha J., Sherina S., Dija S., Thomas K. (2014). Volatile Internet Evidence Extraction from Windows Systems. Proc. IEEE Intl. Conf. on Computational Intelligence and Computing Research, Coimbatore, India, September.

Pan L., Savoldi A., Gubian P., and Batten L. (2008). Measure of Integrity Leakage in Live Forensic Context. Proc. Intl. Conf. on Intelligence Information Hiding and Multimedia Signal Processing, August, Harbin, China.

Rekall Team. (2015). Rekall Memory Forensic Framework: About the Rekall Memory Forensic Framework. Retrieved March 13, 2015 from www.rekall-forensic.com/ about.html.

Stuttgen, J., Cohen M. (2013). Anti-Forensic Resilient Memory Acquisition. Digital Investigation, Vol. 10, pp. S105-S115. 
Sutherland, I., Evans, J., Tryfonas, T., Blyth A. (2008). Acquiring Volatile Operating System Data Tools and Techniques. ACM SIGOPS Operating Systems Review, Vol. 42, No. 3, April, pp. 65-73.

Volatility Foundation. (2015). The Volatility Foundation - Open Source Memory Forensics. Retrieved March 13, 2015 from www.volatilityfoundation.org/\#!about/cmf 3.

Vömel, S., Freiling, F. (2012). Correctness, Atomicity, and Integrity: Defining Criteria for Forensically-Sound Memory Acquisition. Digital Investigation, Vol. 9, pp. $125-137$.

Zhang, L., Zhang D., Wang L. (2010) Live Digital Forensics in a Virtual Machine. Proc. IEEE Conf. on Computer Application and System Modeling, Taiyuan, China, October. 